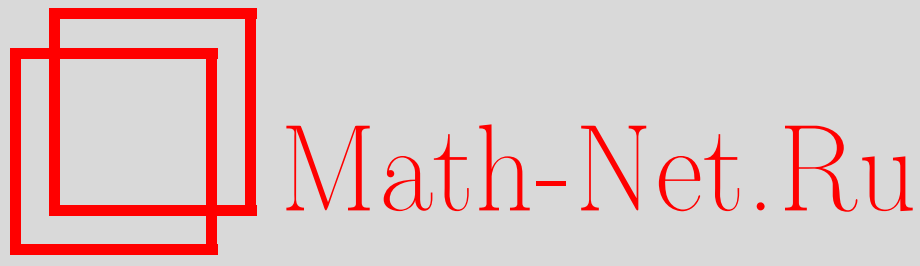

Общероссийский математический портал

Ю. А. Пешкичев, Непрерывность по Кудрявцеву эллиптических отображений, Матем. заметки, 2000, том 68, выпуск 1, 145-146

DOI: https://doi.org/10.4213/mzm930 
Использование Общероссийского математического портала Math-Net.Ru подразумевает, что вы прочитали и согласны с пользовательским соглашением http://www. mathnet.ru/rus/agreement

Параметры загрузки:

IP : 3.85 .7 .115

26 апреля 2023 г., 16:09:13

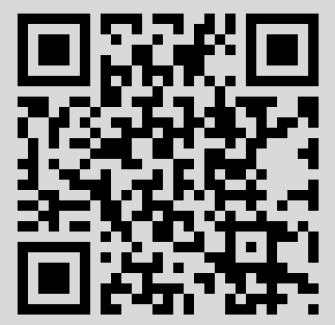




\section{НЕПРЕРЫВНОСТЬ ПО КУДРЯВЦЕВУ ЭЛЛИПТИЧЕСКИХ ОТОБРАЖЕНИЙ}

\section{Ю. А. Пешкичев}

Кроме известной уже непрерывности по Гёльдеру при рассмотрении искажения эллиптических отображений $f: G \rightarrow E^{n}$ открытой области $G \subset E^{n}(n \geqslant 3)$ на гиперповерхностях в области $G$ возникает понятие $(n-1)$-непрерьвности, которое присуще векторной функции

$$
f(x)=\left[f_{1}(x), f_{2}(x), \ldots, f_{m}(x)\right], \quad m \geqslant n-1,
$$

и которое вырождается при $m<n-1$. Поскольку такая непрерьвность проявляется только при ограниченности вариации Кудрявцева эллиптического отображения $f$, естественно назвать ее непрерывностью по Кудрявиеву.

Здесь и в дальнейшем $E^{n}$ - евклидово пространство точек $x=\left(x_{1}, x_{2}, \ldots, x_{n}\right), H_{n-1}$ - мера Хаусдорфа в $E^{n}$ размерности $n-1, B_{n}(x, r)$ - шар в $E^{n}$ с центром в точке $x$ радиуса $r, S_{n-1}(x, r)$ - его граница, $\sigma_{n-1}=H_{n-1}\left(S_{n-1}(0,1)\right)$.

Для гомеоморфизма $f: G \rightarrow E^{n}$ соболевского класса $W_{n}^{1}(G)$ и числа $p \geqslant 1$ рассмотрим $p$-вариацию Кудрявцева [1]

$$
\stackrel{p}{\underset{G}{V} f}=\int_{G}|J f(x)|^{p} d x
$$

где $J f(x)$ - якобиан отображения $f$ в точке $x \in G$, определенный почти всюду в $G$. Пусть $|\nabla f(x)|$ - евк лидова норма матрица Якоби отображения $f$ в точке $x$. Отображение $f: G \rightarrow E^{n}$ класса $W_{n}^{1}(G)$ назьвается әллиптическим [2], если для почти всех $x \in G J f(x)>0$ и

$$
|\nabla f(x)|^{n} \leqslant(n Q)^{n / 2} J f(x)+Q_{1}(x)|f(x)|^{n}+Q_{2}(x),
$$


где $Q \geqslant 1, Q_{1}(x) \geqslant 0, Q_{2}(x) \geqslant 0$ и $Q_{1}(x), Q_{2}(x) \in L_{q}(G), q>1$.

Пусть для $(n-2)$-контура $\Gamma \subset E^{n}$

$$
\operatorname{Vol}_{n-1}(\Gamma)=\inf _{\sigma \in \Sigma} H_{n-1}(\sigma),
$$

где $\Sigma$ - совокупность всех спрямляемых по мере $H_{n-1}\left[3\right.$, с. 272] гиперповерхностей $\sigma$ в $E^{n}$, натянутых на $\Gamma$.

Пусть шар $B_{n}(x, r)$ принадлежит области $G \subset E^{n}, S_{n-2}(x, r, e)$ - диаметральное сечение его границы $S_{n-1}(x, r)$ гиперплоскостью с вектором нормали $e$, проходящей через точку $x$. Рассмотрим $(n-1)$-колебание непрерьвного отображения $f: G \rightarrow E^{n}$ на сфере $S_{n-1}(x, r)$

$$
\operatorname{osc}_{n-1} f(x, r)=\inf _{e \in \Omega} \operatorname{Vol}_{n-1}\left(f\left(S_{n-2}(x, r, e)\right)\right),
$$

где $\Omega$ - совокупность всех единичных векторов $e=x /|x|$.

Для непрерывно дифференцируемого отображения $f: G \rightarrow E^{n}$

$$
\begin{aligned}
& \lim _{r \rightarrow 0} \frac{\operatorname{osc}_{n-1} f(x, r)}{r^{n-1}} \leqslant \sigma_{n-1}|\nabla f(x)|^{n-1}, \\
& \lim _{r \rightarrow 0} \frac{\operatorname{osc}_{n-1} f(x, r)}{r^{\gamma(n-1)}}=0 \quad(0<\gamma<1) .
\end{aligned}
$$

Учитывая эти результаты и конструкцию понятия вариации Кудрявцева [1], непрерьвное отображение $f: G \rightarrow E^{n}$ назовем непрерывным по Кудрявиеву с показателем $\gamma \in(0,1)$, если для всех $x \in G$

$$
\varlimsup_{r \rightarrow 0} \frac{\operatorname{osc}_{n-1} f(x, r)}{r^{\gamma}(n-1)} \leqslant \text { Const }<\infty .
$$

Теорема. Если ограниченное гомеоморфное эллиптическое отобрахсение $f: G \rightarrow E^{n}$ имеет ограниченную $р$-вариацию Кудрявиева при $p=\alpha(n-1) /(n(\alpha-1)), 1<\alpha<2$, $Q_{1}(x), Q_{2}(x) \in L_{p}(G)$, то отображсние $f$ непрерывно по Кудрявчеву с показателем $\gamma=(n-\alpha) /(\alpha(n-1))$.

\section{СПИСОК ЦИТИРОВАННОЙ ЛИТЕРАТУРЫ}

1. Кудрявцев Л. Д. // УМН. 1955. Т. 10. № 2. С. 167-174. 2. Осколков А. П. // Тр. МИАН. 1966. Т. 92. С. 182-191. 3. Федерер Г. Геометрическая теория меры. М.: Наука, 1987. 4. Пешкичев Ю. А. // Матем. заметки. 1994. Т. 55. №1. С. 74-78. 5. Gehring F. W. // Acta Math. 1973. V. 30. P. 265-277. 